\title{
Long-term follow-up and clinical course of a rare case of von Hippel-Lindau disease: A case report and review of the literature
}

\author{
YU ZOU ${ }^{1}$, JINGJING XU ${ }^{2}$ and MINMING ZHANG ${ }^{2}$ \\ ${ }^{1}$ Department of Radiology, Women's Hospital, School of Medicine, Zhejiang University, Hangzhou, Zhejiang 310006; \\ ${ }^{2}$ Department of Radiology, The Second Affiliated Hospital, School of Medicine, Zhejiang University, \\ Hangzhou, Zhejiang 310009, P.R. China
}

Received March 2, 2015; Accepted March 15, 2016

DOI: $10.3892 / \mathrm{ol} .2016 .4387$

\begin{abstract}
Hippel-Lindau (VHL) disease is an autosomal dominantly inherited neoplastic syndrome that increases susceptibility to a variety of benign and malignant neoplasms. It has been well documented that, despite complete excision of initial neoplasms, VHL patients may develop further lesions, in some cases many years after the initial diagnosis. Therefore, the varied and complex clinical manifestations and radiological findings of VHL are of interest. The current study is the first to describe a case of VHL disease with lateral ventricular hemangioblastomas and subsequent pancreatic neuroendocrine tumor, bilateral renal cysts and renal cell carcinoma. The complete clinical course, radiological findings, including ultrasound, computed tomography and magnetic resonance imaging (MRI), and associated pathological findings are presented. Radiology is critical in the accurate diagnosis, treatment and follow-up of VHL. Continuous close and long-term follow-up with radiological examination, particularly MRI, must be conducted in patients with VHL disease. To the best of our knowledge, the current case is a rare phenomenon that has not yet been described in the English literature.
\end{abstract}

\section{Introduction}

von Hippel-Lindau (VHL) disease is an autosomal dominantly inherited neoplastic syndrome that increases susceptibility to a variety of benign and malignant neoplasms, including retinal and central nervous system (CNS) hemangioblastomas,

Correspondence to: $\mathrm{Dr}$ Minming Zhang, Department of Radiology, The Second Affiliated Hospital, School of Medicine, Zhejiang University, 88 Jiefang Road, Hangzhou, Zhejiang 310009, P.R. China

E-mail: cjr.zhangminming@vip.163.com

Key words: von Hippel-Lindau, central nervous system, hemangioblastoma, pancreatic neuroendocrine tumor, renal cell carcinoma clear cell renal cell carcinomas (RCCs), pheochromocytomas, pancreatic neuroendocrine tumors (PanNETs) and endolymphatic sac tumors (1). In addition, renal cysts, pancreatic cysts or cystadenomas, and epididymal or broad ligament cystadenomas may also occur (2).

The identification of the VHL gene has allowed for the development of molecular genetic diagnosis of VHL disease by direct mutation analysis (3). It has been well documented that VHL patients may develop further de novo lesions, occasionally many years after the initial diagnosis, despite the complete excision of initial neoplasm (4). Thus, long-term follow-ups for patients with VHL disease are necessary. Clinical courses are occasionally complicated, and are of interest because VHL involves multiple organs but results in organ-specific tumors (5). The current study reports the case of a patient with VHL disease complicated with lateral ventricular hemangioblastomas and, subsequently, PanNET, bilateral renal cysts and clear cell RCC.

\section{Case report}

A 17-year-old male presented to The Second Affiliated Hospital of Zhejiang University School of Medicine (Hangzhou, China) in September 2007, with a progressive headache and dizziness of three months duration. He had experienced no previous medical problems except for a single episode of generalized convulsion 2 months prior to admission. The neurological examination was normal. Magnetic resonance imaging (MRI) of the brain was performed using a 1.5-T MR imaging system (Signa; GE Healthcare, Milwaukee, WI, USA) and revealed a mass near the septum pellucidum, and a subependymal nodule at a same level in the right lateral ventricle. The mass near the septum pellucidum was hypointense on T1-weighted images and hyper-isointense on T2-weighted images relative to the normal white matter. Gadolinium-enhanced T1-weighted MRI demonstrated a solid, rounded, intraventricular tumor of $2 \mathrm{~cm}$ in diameter, with strong contrast enhancement. However, the subependymal nodule was hyper-isointense on T1-weighted images and hypointense on T2-weighted images, which indicated hemorrhage. The nodule exhibited slight contrast enhancement (Fig. 1A-D). 

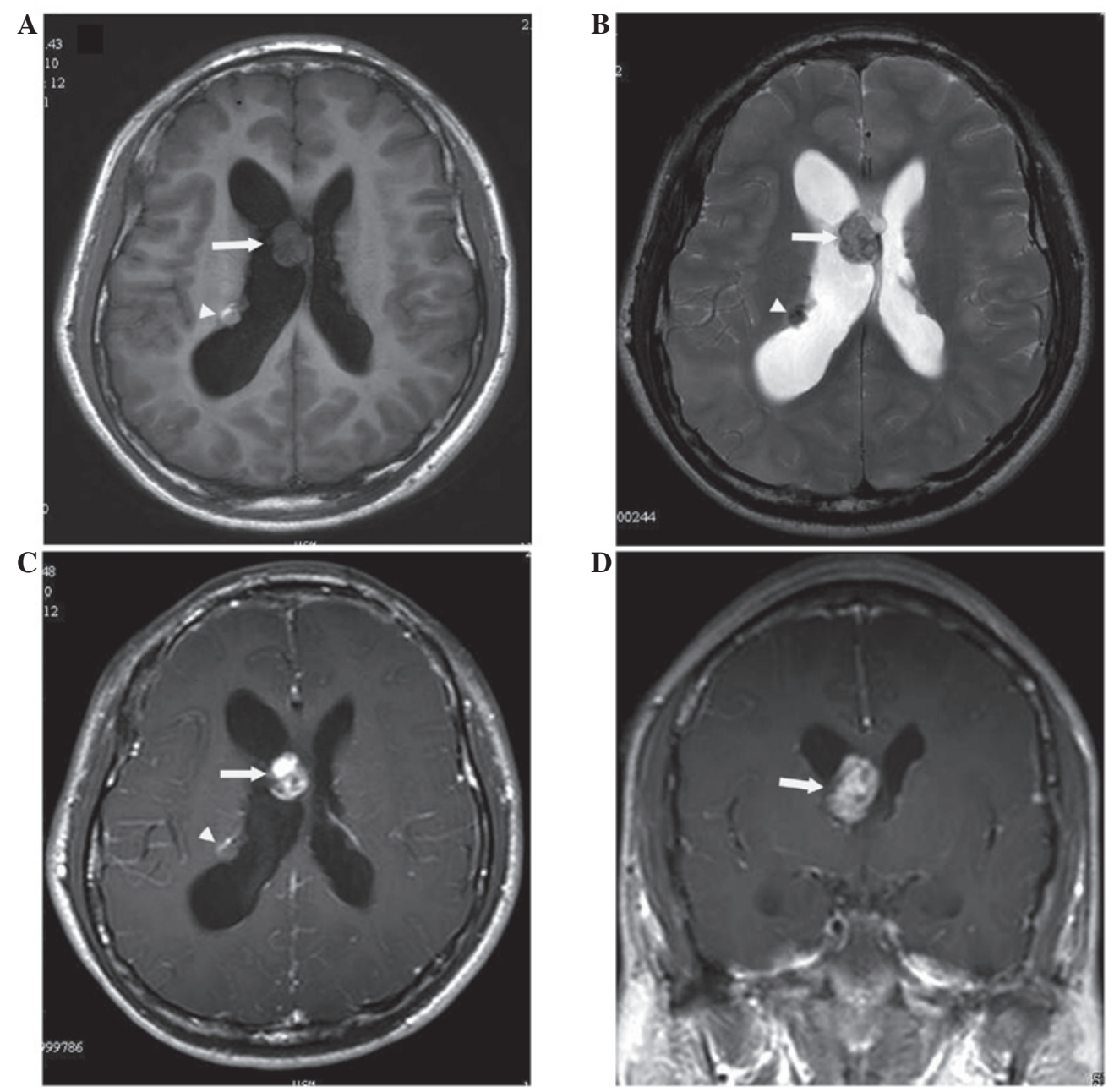

Figure 1. Magnetic resonance imaging scans of 17-year-old male who presented with a progressive headache and dizziness of three months duration. Axial unenhanced (A) T1-weighted and (B) T2-weighted images revealed a mass near the septum pellucidum (arrow) and a subependymal nodule (arrow head) in the right lateral ventricle. The nodule was hyper-isointense on T1-weighted images and hypointense on T2-weighted images, which indicated hemorrhage. (C) Axial and (D) coronal contrast-enhanced fat-suppressed T1-weighted images revealed that the mass (arrow) exhibited strong enhancement, whilst the nodule (arrow head) exhibited slight enhancement.

In September 2007, the patient underwent a right-sided parietal craniotomy. The mass and subependymal nodule were completely excised via a trans-sulcal approach through the superior parietal lobule. Grossly, the mass was cherry-red, foam-like and highly vascular. However, the subependymal nodule was brownish, slightly firm and was adherent to the ventricular ependyma. The resected specimen was fixed with $4 \%$ neutral formaldehyde, followed by conventional dehydration, paraffin embedding, sectioning, and hematoxylin and eosin (HE) staining. Histopathological examination of both lesions revealed tissue composed of many small blood vessels separated by numerous polygonal stromal cells, with lightly stained cytoplasm. Additionally, hemorrhagic foci were observed within the tissue of the subependymal nodule, in accordance with the MRI findings (Fig. 1). Immunohistochemical staining was performed using the EnVision two-step method. Antibodies to CD34 (mouse monoclonal 1:100 dilution), chromogranin A (mouse monoclonal 1:100 dilution), synaptophysin (mouse monoclonal 1:50 dilution) and Ki67 (mouse monoclonal 1:50 dilution), which were all purchased from Funakoshi, Co., Ltd. (Tokyo, Japan). Immunohistochemical staining revealed strong immunopositivity in several stromal cells for vimentin, epithelial membrane antigen and neuron specific enolase. The intercapillary component was immunopositive for the endothelial cell marker CD34. Histopathological examination and immunohistochemical staining confirmed that the two lesions were typical hemangioblastomas (Fig. 2A-B) (6).

Postoperatively, the patient experienced a transient sensory aphasia, which recovered fully within 2 weeks; the subsequent course was uneventful. According to clinical criteria (7), a suspected diagnosis of VHL disease was established for this patient. Thus, additional systematic enquiry and examinations associated with VHL disease were performed, including funduscopic examination, abdominal spiral computed tomography (CT; LightSpeed VCT 64; GE Healthcare, Pittsburgh, PA, USA), magnetic resonance (MR) scanning of the spinal cord, and 24-h urinary analysis for vanillylmandelic acid, metanephrines and total catecholamines. The results of the monitoring workup were negative, with the exception of multiple bilateral renal cysts that were visualized by abdominal CT examination. No family history of specific diseases was reported. A mutation analysis was completed; however, following polymerase chain reaction and sequencing of three exons of the VHL gene, no variation was detected. 

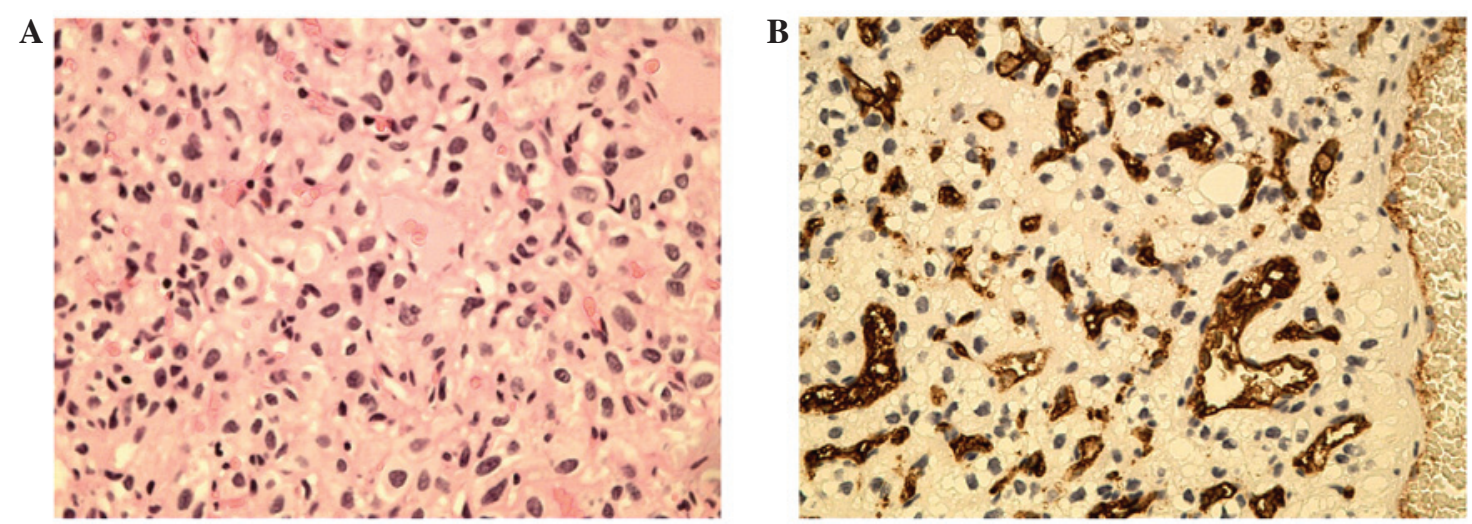

Figure 2. (A) Histopathology of the tissue from the mass in the right lateral ventricle. Numerous small blood vessels with a few foam cells, fat-like cells and fibroblasts were observed (hematoxylin and eosin; magnification, x400). (B) Immunohistochemical assay of the tissues from the mass near the septum pellucidum in the right lateral ventricle (magnification, $\mathrm{x} 400$ ). Many immunopositive intercapillary components were stained by the endothelial cell marker CD34.
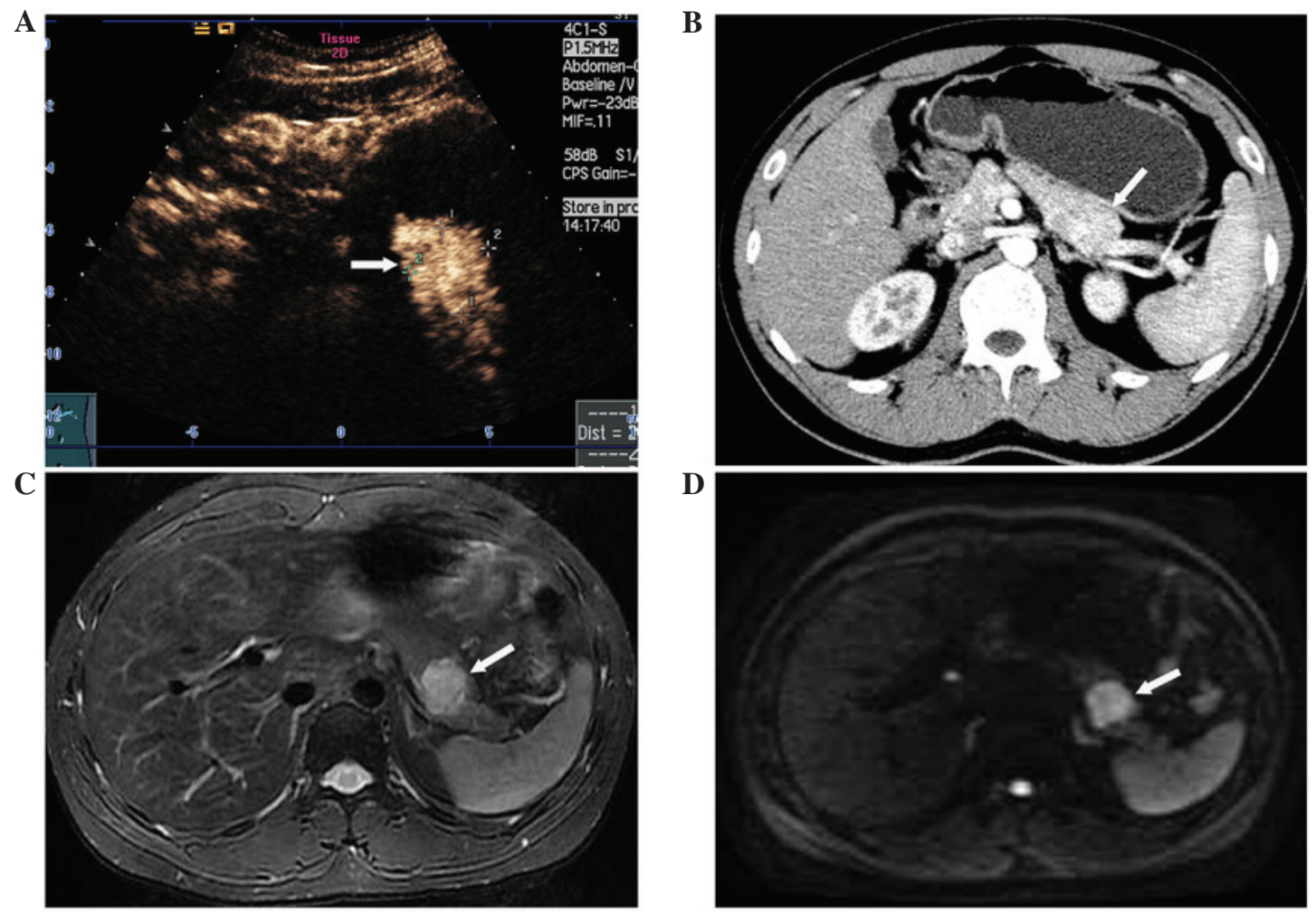

Figure 3. (A) Contrast-enhanced ultrasonography revealed a pancreatic solid tumor of $3 \mathrm{~cm}$ in diameter with marked contrast enhancement (arrow). (B) Enhanced abdominal computed tomography scans failed to delineate the pancreatic lesion clearly, except for a focal swelling and contour-deformation of the distal body of the pancreas (arrow). (C) The pancreatic lesion (arrow) was hyperintense relative to normal pancreatic tissue on fat-suppressed T2-weighted MRI. (D) A homogeneous, 3-cm, well-defined mass with high signal intensity (arrow) was delineated clearly on diffusion-weighted $\mathrm{MRI}\left(\mathrm{b}=1,000 \mathrm{sec} / \mathrm{mm}^{2}\right)$ (arrow). MRI, magnetic resonance imaging.

At $10 \mathrm{pm}$ on 12th December 2013, the patient was readmitted to the emergency department of The Second Affiliated Hospital of Zhejiang University School of Medicine due to an attack of syncope and loss of consciousness without convulsion of the limbs. Emergency laboratory data confirmed hypoglycemic shock (plasma glucose level, $1.18 \mathrm{mmol} / \mathrm{l}$; normal fasting glucose range, 3.89-6.1 $\mathrm{mmol} / \mathrm{l})$. The patient's symptoms were alleviated significantly following the administration of glucose via intravenous injection. Subsequently, pancreatic exploration was conducted, and pancreatic routine ultrasonography suggested a hypoechoic lesion of $3 \mathrm{~cm}$ in diameter on the distal body of the pancreas. Contrast-enhanced ultrasonography with enhancement material, consisting of SonoVue (Bracco, Milan, Italy) diluted with $5 \mathrm{ml}$ of normal sodium, was performed and revealed a pancreatic solid tumor with marked contrast enhancement (Fig. 3A). During ultrasound examination (SSA-790A; Toshiba Medical Systems, Tokyo, Japan) with a convex probe (PVT-375BT; 3.75-MHz center frequency), multiple small cysts and multiple small hypoechoic solid lesions were 

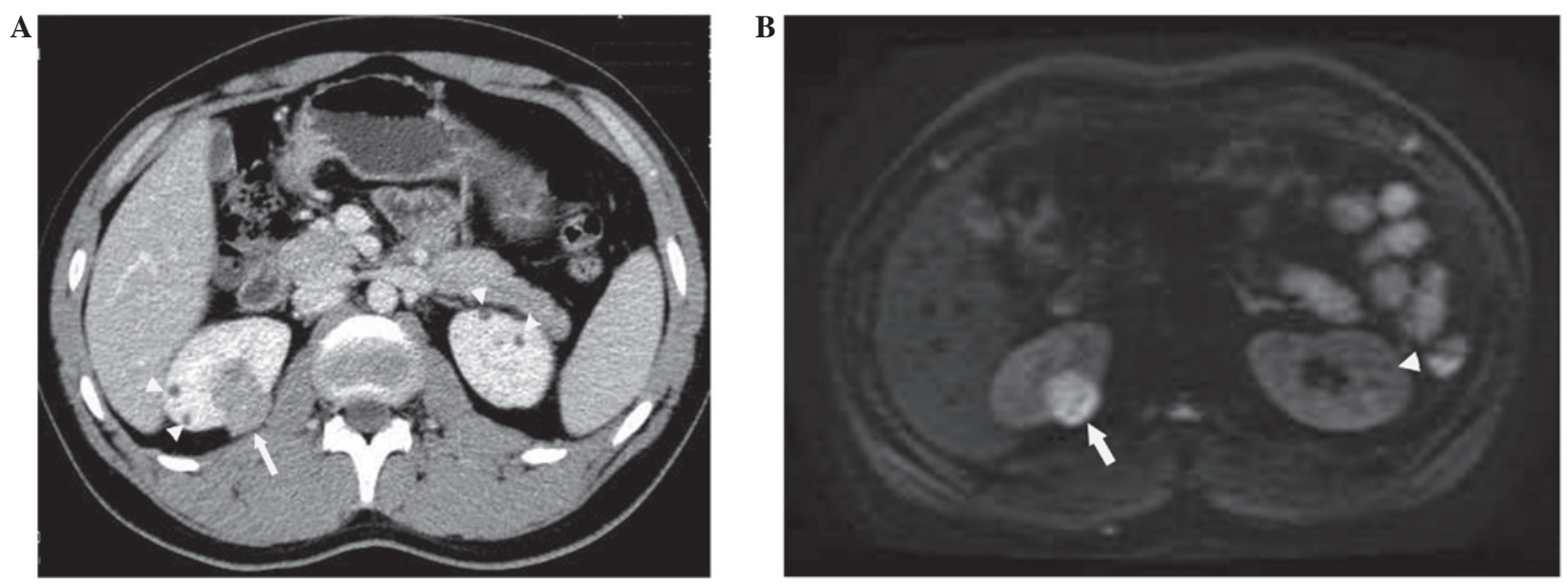

Figure 4.(A) Contrast computed tomography scanning of the kidneys revealed multiple cystic lesions (arrow head) and solid lesions (arrow). (B) Diffusion-weighted magnetic resonance imaging of the kidney revealed hypointensity for all cystic lesions, and hyperintensity for solid lesions.

detected in the bilateral kidneys. Thus, abdominal CT and MRI were performed to further characterize these complex lesions. Unenhanced and enhanced abdominal CT scans failed to delineate the pancreatic lesion clearly, except for a focal swelling and contour-deformation of the distal body of the pancreas (Fig. 3B). However, all of the pancreatic and renal lesions (cystic and solid) were delineated clearly using a Signa HDx 3.0T MR scanner (GE Healthcare, Milwaukee, WI, USA). The pancreatic lesion was hyperintense relative to normal pancreatic tissue on fat-suppressed $\mathrm{T} 2$-weighted sequences, and hypointense on fat-suppressed unenhanced T1-weighted sequences (Fig. 3C). Additional diffusion-weighted imaging (DWI) was also acquired using a single-shot, echo-planar-imaging sequence with short tau inversion recovery-based fat suppression (Fig. 3D). Water diffusion was measured at b-values of 0 and $1,000 \mathrm{sec} / \mathrm{mm}^{2}$. A homogeneous, well-defined mass of $3 \mathrm{~cm}$ in diameter, which had high signal intensity and was located in the distal body of the pancreas, was delineated more clearly on DWI $\left(b=1,000 \mathrm{sec} / \mathrm{mm}^{2}\right)$, and there were decreased apparent diffusion coefficients (ADCs) on the ADC map as compared with the adjacent normal tissue of the pancreas. Regions of interest (ROI) were drawn within the tumor and the normal-appearing pancreas. The measured ADC values were $0.610 \times 10^{-3} \mathrm{~mm}^{2} / \mathrm{sec}$ in the tumor and $1.143 \times 10^{-3} \mathrm{~mm}^{2} / \mathrm{sec}$ in the normal-appearing pancreas. On dynamic gadolinium-based contrast-enhanced and fat-suppressed T1-weighted images, the lesion exhibited early enhancement during the arterial phase and progressive fill-in and enhancement during the delayed phase.

In accordance with the ultrasonographic findings, abdominal CT imaging with contrast revealed multiple bilateral cystic and solid renal lesions of varying sizes (range, 2-20 mm). The solid lesions had marked contrast enhancement, whereas the cystic lesions did not (Fig. 4A). Similarly, renal MRI revealed multiple cystic lesions and multiple solid lesions in both kidneys. The multiple cystic lesions ( 8 in the right kidney, $2-10 \mathrm{~mm}$ in diameter; and 6 in the left kidney, 2-8 $\mathrm{mm}$ in diameter) exhibited homogeneous hyperintensity on $\mathrm{T} 2$-weighted images and hypointensity on fat-suppressed T1-weighted images, and no enhancement on contrast-enhanced and fat-suppressed T1-weighted images. The solid lesions (4 in the right kidney, $3-20 \mathrm{~mm}$ in diameter; and 3 in the left kidney, 2-10 $\mathrm{mm}$ in diameter) were hypointense on T1- and T2-weighted images and had strong enhancement on contrast-enhanced and fat-suppressed T1-weighted images. DWI with a b-value of $1,000 \mathrm{sec} / \mathrm{mm}^{2}$ revealed that the signal intensities were reduced for all cystic lesions and increased considerably for all solid lesions (Fig. 4B). ADC maps confirmed these findings, yielding high values $\left(0.97 \times 10^{-3} \mathrm{~mm}^{2} / \mathrm{sec}\right)$ for all cystic lesions and low values $\left(0.57 \times 10^{-3} \mathrm{~mm}^{2} / \mathrm{sec}\right)$ for all solid lesions. These data suggested a preoperative diagnosis of PanNET and multiple bilateral renal cysts and RCCs.

On 7th January 2014, due to the malignant potential of the PanNET and the multifocal spread of the bilateral renal tumors, simultaneous distal splenopancreatectomy and enucleation of the bilateral renal tumors were performed. A lesion of $3 \mathrm{~cm}$ in diameter within the distal body of the pancreas was identified. The tumor was a well-demarcated, red-brown mass, and its cut surface appeared solid and homogeneous. At histological examination, the tumor was characterized as a well-differentiated PanNET (8); immunohistochemically the tumor exhibited chromogranin A and synaptophysin positivity, and a Ki-67 index $<2 \%$ (Fig. 5A-C).

Intraoperatively, 6 tumors in the right kidney and 5 tumors in left kidney could be reached and were enucleated. The renal vein and inferior vena cava were normal, with no obvious abnormal lymph nodes. Histopathological examination revealed typical clear cell RCC (pT1, cN0, cM0, G2, $\mathrm{R} 0$ ), which contained clear cells with inconspicuous nucleoli forming prominent microcysts (9), in both kidneys (Fig. 5D).

The patient's postoperative course was uneventful and he was discharged on the 10th postoperative day, with a regular follow-up recommended. At follow-up to date, the patient is asymptomatic and investigations have revealed no tumor recurrence or presence of any further masses in any other organ. Informed consent was obtained from the patient. The study was approved by the patient and the Second Affiliated Hospital, School of Medicine, Zhejiang University Service Ethics Committee (Zhejiang, China). 

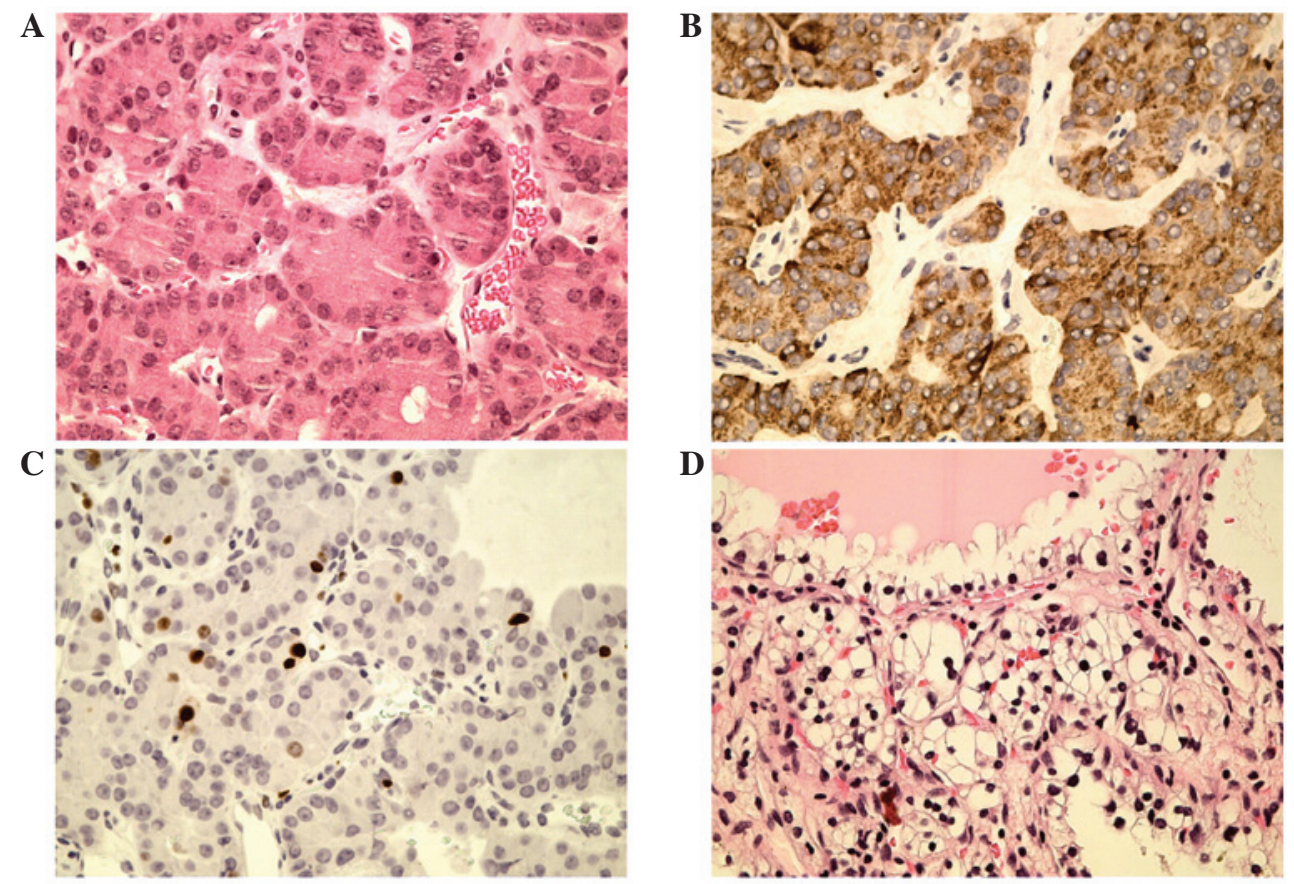

Figure 5. (A-C) At histological examination, the pancreatic tumor was characterized as a well-differentiated pancreatic neuroendocrine tumor: (A) hematoxylin and eosin staining (magnification, x400); (B) positive chromogranin A immunostaining; and (C) Ki-67 index <2\%. (D) The renal tumors had a microscopic appearance typical of clear cell renal cell carcinoma (hematoxylin and eosin staining; magnification, $\mathrm{x} 100$ ).

\section{Discussion}

Hemangioblastomas of the CNS are uncommon, accounting for $2 \%$ of primary CNS tumors $(1,3)$. These tumors may occur sporadically; however, they have been found to be associated with VHL disease in $\sim 30 \%$ of cases. Hemangioblastomas are predominantly detected in the cerebellum, spinal cord or brainstem (in $\sim 83 \%$ of cases, collectively) (2). Less common locations include the leptomeninges and sellar-sphenoid sinus $(4,7,10)$. Supratentorial locations are rare, representing $4 \%$ of sporadic hemangioblastomas and $13 \%$ of those associated with VHL disease (10). Supratentorial intraventricular hemangioblastomas are extremely rare, and only 7 cases (excluding the present case) of lateral ventricular hemangioblastomas have been reported in the English literature to date; 6 were identified to be associated with VHL disease and 1 case was undefined. Thus, the strong possibility of VHL disease should be raised in patients with hemangioblastomas in such unusual locations (10).

In the current case, establishing a preoperative diagnosis of hemangioblastoma of the lateral ventricle was extremely challenging based on imaging findings. The lesions were suspected to be 'subependymal astrocytoma' based on the patient's age and certain imaging features, such as the location of the lesion and the coexistent tumor and subependymal nodule. According to clinical criteria (7), patients with $\geq 2$ CNS hemangioblastomas or 1 CNS hemangioblastoma and a VHL-associated visceral tumor fulfill the criteria for clinical diagnosis without a familial history of VHL disease $(7,11,12)$. Thus, a final diagnosis of VHL disease could be established in the current patient due to the 2 hemangioblastomas and the multiple bilateral cysts.

With respect to the natural history of VHL disease, Wanebo et al (13) reported that VHL-associated tumors have two separate phases of growth, termed rapid and quiescent growth periods, if the patient is observed for a sufficient duration. A rapid growth period is typically followed by a quiescent period that can last $>30$ months. In the present case, 75 months after the initial surgery, the PanNET was identified. Furthermore, the cystic masses in the kidneys subsequently developed into RCCs.

Pancreatic tumors or cysts develop in $35-77 \%$ of VHL patients in the majority of case series, with most of the cysts being benign (14). Pancreatic tumors include cystadenomas $(12 \%)$, hemangioblastomas $(<1 \%)$, adenocarcinomas $(<1 \%)$ and PanNETs (9-17\%) (15). PanNETs are tumors with an abundant blood supply, and the pancreas is an organ with high perfusion. Therefore, on contrast CT scanning, pancreatic tumors and the organ itself exhibit simultaneously enhancement. The difference in density is not as obvious as for pancreatic carcinoma, which has a poor blood supply. Large PanNETs sometimes appear only as a change in the contour of the pancreas, as in the current case, whilst small PanNETs are easily missed at diagnosis. MRI with superior tissue contrast is able to show PanNETs better, particularly on DWI sequences when detecting small lesions. Thus, MRI (and most importantly DWI) is essential for monitoring the pancreas in VHL patients.

PanNETs associated with VHL disease are typically nonfunctional and are located throughout the pancreas. These tumors are often asymptomatic; however, they can cause pancreatitis or a mass-related pain (16). Patients can occasionally present hypoglycemic symptoms, such as in the present case, due to neoplastic expansion or local invasion. This would be an indication for surgical treatment. Resection is recommended for tumors $>3 \mathrm{~cm}$, and the patients should be followed up closely (17). 
VHL-associated pancreatic lesions are always associated with renal lesions (18). Patients with VHL disease are at high risk of developing multiple renal cysts and RCCs, which occurs in around two-thirds of patients (19). The most frequent histological form of RCC is clear cell carcinoma which, in the majority of cases, tends to be multifocal and involve both kidneys. Although renal cysts may be benign, they are considered premalignant lesions; RCCs may arise from cystic or non-cystic renal parenchyma (20). The primary therapeutic approach is surgery, aiming to preserve the maximal amount of renal parenchyma (21). The onset of renal tumors represents the predominant cause of mortality in patients with VHL syndrome $(\sim 70 \%)$; thus, early diagnosis followed by a possible partial nephrectomy in order to avoid dialysis and renal transplantation is extremely important $(21,22)$.

In conclusion, the current case suggests that a high degree of suspicion for VHL disease should be raised in patients with hemangioblastomas in supratentorial locations, particularly when intraventricular. In addition, follow-up of VHL patients must not be discontinued after their initial diagnosis and treatment, even if they experience symptom-free periods. Furthermore, MR examination, particularly DWI sequences, is valuable when abdominal organs, such as the pancreas or kidneys, are involved. In summary, the current study emphasizes the importance of close follow-ups with radiological examination of CNS hemangioblastoma and other organs subsequent to the initial presentation of CNS hemangioblastoma associated with VHL disease.

\section{References}

1. Maher ER and Kaelin WG Jr: Von Hippel-Lindau disease. Medicine (Baltimore) 76: 381-391, 1997.

2. Kaelin WG: Von Hippel-Lindau disease. Annu Rev Pathol 2: 145-173, 2007.

3. Latif F, Tory K, Gnarra J, Yao M, Duh FM, Orcutt ML, Stackhouse T, Kuzmin I, Modi W, Geil L, et al: Identification of the von Hippel-Lindau disease tumor suppressor gene. Science 260: 1317-1320, 1993.

4. Kaelin WG Jr: Molecular basis of the VHL hereditary cancer syndrome. Nat Rev Cancer 2: 673-682, 2002.

5. Gossage L, Eisen T and Maher ER: VHL, the story of a tumour suppressor gene. Nat Rev Cancer 15: 55-64, 2014.

6. Sundaram C, Rammurti S, Reddy JJ, Prasad SS and Purohit AK: Hemangioblastoma: A study of radiopathologic correlation. Neurol India 51: 373-375, 2003.
7. Lonser RR, Glenn GM, Walther M, Chew EY, Libutti SK, Linehan WM and Oldfield EH: Von Hippel-Lindau disease. Lancet 361: 2059-2067, 2003.

8. Batcher E, Madaj P and Gianoukakis AG: Pancreatic neuroendocrine tumors. Endocr Res 36: 35-43, 2011

9. Lee C, Park JW, Suh JH, Nam KH, et al: Histologic variations and immunohistochemical features of metastatic clear cell renal cell carcinoma. Korean J Pathol 47: 426-432, 2013.

10. Takeuchi S and Takasato Y: Supratentorial intraventricular hemangioblastomas. Acta Neurol Belg 111: 353-356, 2011.

11. Johnston LB, Chew SL, Lowe D, Reznek R, Monson JP and Savage MO: Investigating familial endocrine neoplasia syndromes in children. Horm Res 55 (Suppl 1): S31-S35, 2001.

12. Gaal J and de Krijger RR: Neuroendocrine tumors and tumor syndromes in childhood. Pediatr Dev Pathol 13: 427-441, 2010.

13. Wanebo JE, Lonser RR, Glenn GM and Oldfield EH: The natural history of hemangioblastomas of the central nervous system in patients with von Hippel-Lindau disease. J Neurosurg 98: 82-94, 2003.

14. Tamura K, Nishimori I, Ito T, et al: Diagnosis and management of pancreatic neuroendocrine tumor in von Hippel-Lindau disease. World J Gastroenterol 16: 4515-4518, 2010.

15. Hammel PR, Vilgrain V, Terris B, Penfornis A, Sauvanet A, Correas JM, Chauveau D, Balian A, Beigelman C, O'Toole D, et al: Pancreatic involvement in von Hippel-Lindau disease. The Groupe Francophone d'Etude de la Maladie de von Hippel-Lindau. Gastroenterology 119: 1087-1095, 2000.

16. Chetty R, Kennedy M, Ezzat S and Asa SL: Pancreatic endocrine pathology in von Hippel-Lindau disease: An expanding spectrum of lesions. Endocr Pathol 15: 141-148, 2004.

17. Blansfield JA, Choyke L, Morita SY, Choyke PL, Pingpank JF, Alexander HR, Seidel G, Shutack Y, Yuldasheva N, Eugeni M, et al: Clinical, genetic and radiographic analysis of 108 patients with von Hippel-Lindau disease (VHL manifested by pancreatic neuroendocrine neoplasms (PNETs). Surgery 142: 814-818; discussion 818.e1-e2, 2007.

18. Mukhopadhyay B, Sahdev A, Monson JP, Besser GM, Reznek RH and Chew SL: Pancreatic lesions in von Hippel-Lindau disease. Clin Endocrinol (Oxf) 57: 603-608, 2002.

19. Chetty R, Kennedy M, Ezzat S and Asa SL: Pancreatic endocrine pathology in von Hippel-Lindau disease: An expanding spectrum of lesions. Endocr Pathol 15: 141-148, 2004.

20. Nickerson ML, Jaeger E, Shi Y, Durocher JA, Mahurkar S, Zaridze D, Matveev V, Janout V, Kollarova H, Bencko V, et al: Improved identification of von Hippel-Lindau gene alterations in clear cell renal tumors. Clin Cancer Res 14: 4726-4734, 2008.

21. Patard JJ, Shvarts O, Lam JS, Pantuck AJ, Kim HL, Ficarra V, Cindolo L, Han KR, De La Taille A, Tostain J, et al: Safety and efficacy of partial nephrectomy for all T1 tumors based on an international multicenter experience. J Urol 171: 2181-2185; quiz $2435,2004$.

22. Bratslavsky G, Liu JJ, Johnson AD, Sudarshan S, Choyke PL, Linehan WM and Pinto PA: Salvage partial nephrectomy for hereditary renal cancer: Feasibility and outcomes. J Urol 179: 67-70, 2008. 